\title{
Surgeons' approach toward clinical nutrition: A survey-based study
}

\author{
Bahadır Osman Bozkırlı1, Rıza Haldun Gündoğdu², Soner Akbaba², Turgay Sayın², Pamir Eren Ersoy ${ }^{3}$
}

\begin{abstract}
Objective: Although many surgical patients face postoperative problems due to a poor nutritional status, there is evidence that many cases of malnutrition still go unnoticed and untreated in surgical wards. This study aims to define the current attitudes of surgeons toward nutritional screening and support.

Material and Methods: A questionnaire with 13 questions was e-mailed to 1500 surgeons. Cross-queries were made over the responses.

Results: The response rate was $20.9 \%$. Most of the respondents (89.5\%) implemented nutritional screening. However, only $24.6 \%$ of these surgeons screened every patient for malnutrition. The time to initiate nutritional support varied among respondents, and only $25.5 \%$ started nutritional support early enough prior to surgery. Only $9.9 \%$ of respondents implemented evidence based practices for preoperative fasting, and $21.2 \%$ preferred immunonutrition products for patients undergoing major abdominal surgery for cancer. The responses of surgeons, who participated in at least one scientific meeting on nutrition per year, were more coherent with the nutrition guidelines.

Conclusions: The results of this study reveal that the awareness and knowledge of clinical nutrition need improving amongst surgeons. To increase this awareness and knowledge, continuous learning throughout their career seems essential.

Keywords: Surgeon, malnutrition, nutritional screening, nutrition support, clinical nutrition, awareness
\end{abstract}

\section{INTRODUCTION}

It is a well-known fact that surgical patients with a suboptimal nutritional status have impaired wound healing, impaired immune responses, increased organ dysfunction, delayed recovery, and increased morbidity and mortality $(1,2)$. However, even though the prevalence of malnutrition is high and may exceed $60 \%$ in patients undergoing gastrointestinal or major elective surgeries, many cases of malnutrition probably go unnoticed and untreated in surgical wards (3-6). As the attending specialist, the surgeon must organize nutritional screening and treatment of the patients in a surgical clinic. This necessitates surgeons to have sufficient knowledge of nutrition and to be very sensitive about the nutritional status of their patients. Unfortunately, limited data on the degree of attention paid to this important subject by surgeons suggests that the awareness of nutritional principles may be insufficient among this group of clinicians (5-7). This study displays the results of a survey designed to define the current attitudes of Turkish surgeons toward nutritional screening and support.

This study was presented at the XVI. Annual Meeting of the European Society of Surgery, 22-24 November 2012, Istanbul, Turkey.

'Department of General Surgery, Ankara Training and Research Hospital, Ankara, Turkey ${ }^{2}$ Department of General Surgery, Ankara Atatürk Training and Research Hospital, Ankara, Turkey

${ }^{3}$ Department of General Surgery, Güven Hospital, Ankara, Turkey

Address for Correspondence Bahadır Osman Bozkırlı e-mail: bbozkirli@gmail.com

Received: 20.04 .2016 Accepted: 03.06.2016

CCopyright 2017 by Turkish Surgical Association

Available online at www.turkjsurg.com

\section{MATERIAL AND METHODS}

A questionnaire consisting of 13 multiple choice questions was designed by the study authors to investigate the surgeons' approaches to perioperative nutritional screening and therapy of the general surgery patient (Appendix). This survey was e-mailed to 1500 general surgeons, all of whom had already finished the training program in surgery and were working in different hospitals in the Turkish Republic. A cover letter that stood for an informed consent was also attached to this e-mail explaining the purpose of this project and assuring the participants of anonymity.

Since this study was based on a survey answered by doctors, no approval was obtained from any ethics committees; however, the study was conducted according to the Helsinki Declaration.

Only the surgeons who stated that they screened the nutritional status of their patients were asked to answer the questions from 5 to 11 as it was essential to take part in nutritional screening in daily clinical practice to answer these questions.

The answers of each surgeon were stored in a database and cross-queries were made over these responses. The surgeons who were working in state hospitals were compared with the surgeons working in teaching 
hospitals. Further, the answers of respondents were compared according to their experience and the number of scientific meetings on clinical nutrition attended by them in one year.

\section{Statistical Analysis}

Chi-Square test (Pearson's Chi-square, continuity correction, and Fisher's exact test) was used to determine association between groups for categorical variables. Descriptive statistics were presented as frequencies and percentages. A p-value less than 0.05 was accepted as statistically significant. Data analysis was performed using Statistical Package for Social Sciences 15.0 (SPSS Inc.; Chicago, IL, USA) software package.

\section{RESULTS}

Three hundred and fourteen surgeons (20.9\%) responded to the questionnaire. Only the first question was answered by all participants. The ratios are given according to the number of the responders of each question. Fifty-two of the surgeons who responded to this survey (16.6\%) had been working for less than 5 years, and 262 (83.4\%) had been working for more than 5 years. One hundred and eighty-six surgeons were working in teaching hospitals (62.6\%), and 111 (37.4\%) were working in state hospitals. This question was not answered by 17 surgeons. The number of surgeons who did not attend any scientific meetings on nutrition in a year was found to be 116 (37.4\%), whereas that of those who attended 1-2 and more than 2 meetings in a year was 156 (50.3\%) and 38 (12.3\%), respectively. The number of surgeons who screened the nutritional status of their patients was 280 (89.5\%). Thirty-three surgeons (\%10.5) stated that they did not practice nutritional screening in their daily clinical routine.

The surgeons working for less than 5 years had a higher tendency to screen the nutritional status of their patients when compared with the surgeons working for more than 5 years. However, this tendency was statistically insignificant $(p=0.140)$ (Figure 1$)$. The surgeons who participated in at least one scientific meeting on nutrition in a year had a significantly higher tendency to screen the nutritional status of their patients than those who did not participate in any meetings $(p<0.001)$ (Figure 2$)$.
Surgeons working in teaching hospitals had a significantly higher participation in at least one scientific meeting on nutrition in a year when compared with the surgeons working in state hospitals $(p<0.001)$ (Figure 3$)$. Statistical analysis did not reveal a significant relationship between participation in scientific meetings and the duration of surgical experience $(p=0.889)$ (Figure 4).

Significantly more surgeons working in teaching hospitals screened the nutritional status of their patients $(p=0.001)$.

When the respondents who screened for nutritional status were asked which of their patients they screened for malnutrition, $24.6 \%$ answered "all of them". The rest of the participants stated that they only screened the patients whom "they thought to be at nutritional risk through inspection" (41.1\%) or who were undergoing a major surgery (34.4\%). The most frequently used nutritional screening technique was laboratory tests $(29.1 \%)$, followed by subjective global assessment (24.5\%), combined use of different screening systems (23.0\%), Nutritional Risk Screening 2002 (19.5\%), and anthropometric assessment systems (3.9\%). When a patient was determined to be under severe nutritional risk, $20.9 \%$ of these participants initiated nutritional support (NS) at 3-4 days prior to surgery, $53.6 \%$ initiated it at 5-7 days, and $25.5 \%$ initiated it at $10-14$ days. The most common method used by the participants to calculate caloric need was to multiply the weight of the patient in kilograms with $25-30 \mathrm{kcal} / \mathrm{kg}$, according to the patients' current clinical status (43.7\%). This was followed by the use of the Harris-Benedict Formula (36.1\%). One hundred and forty-two surgeons $(51.1 \%$ of those who screened their patients) stated that in the postoperative period, they always continued NS in the patients whom they gave preoperative NS. One hundred and thirty-five (48.6\%) surgeons stated that they continued NS only in the patients who could not fulfill their nutritional needs from oral intake. After discharging from the hospital, 68.8\% of the surgeons who practiced nutritional screening recommended the use of oral or enteral NS products to the patients who received support during their hospital stay.

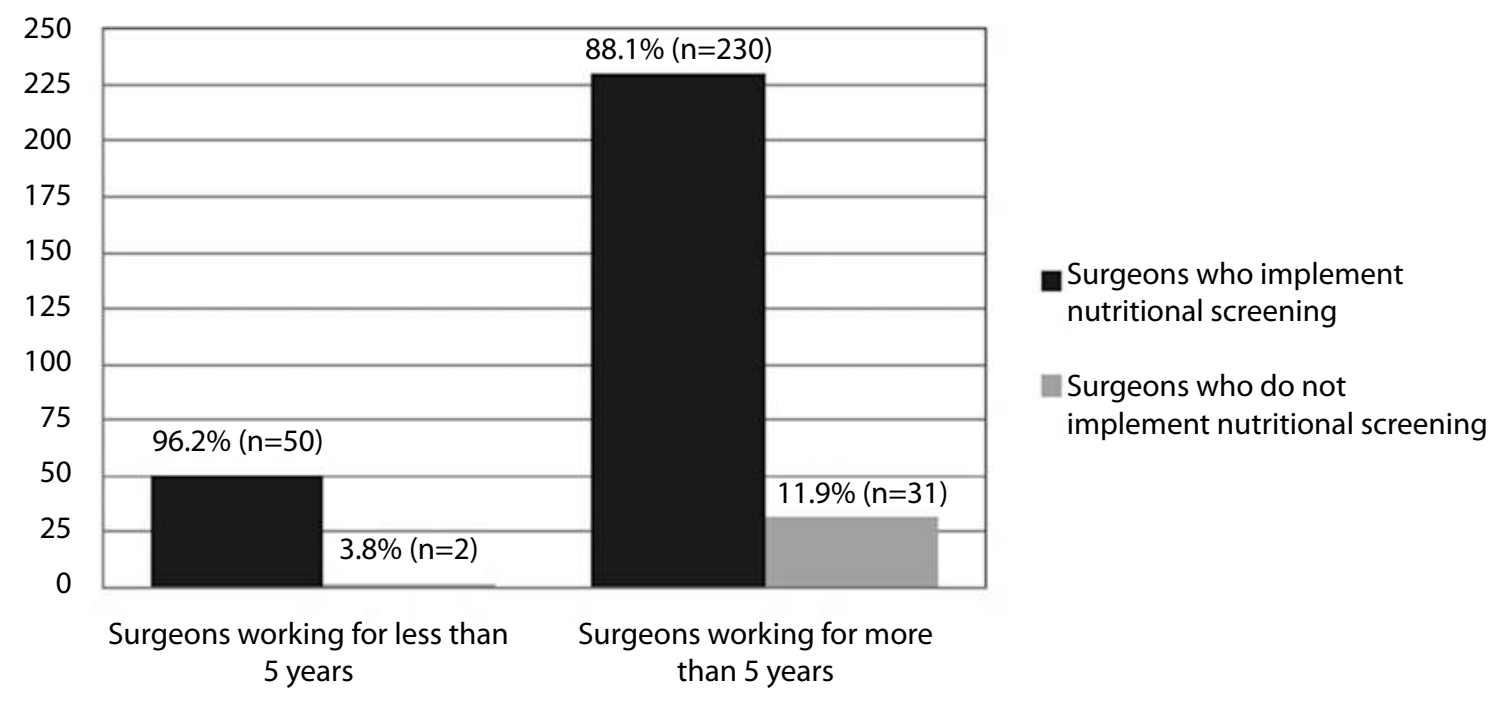




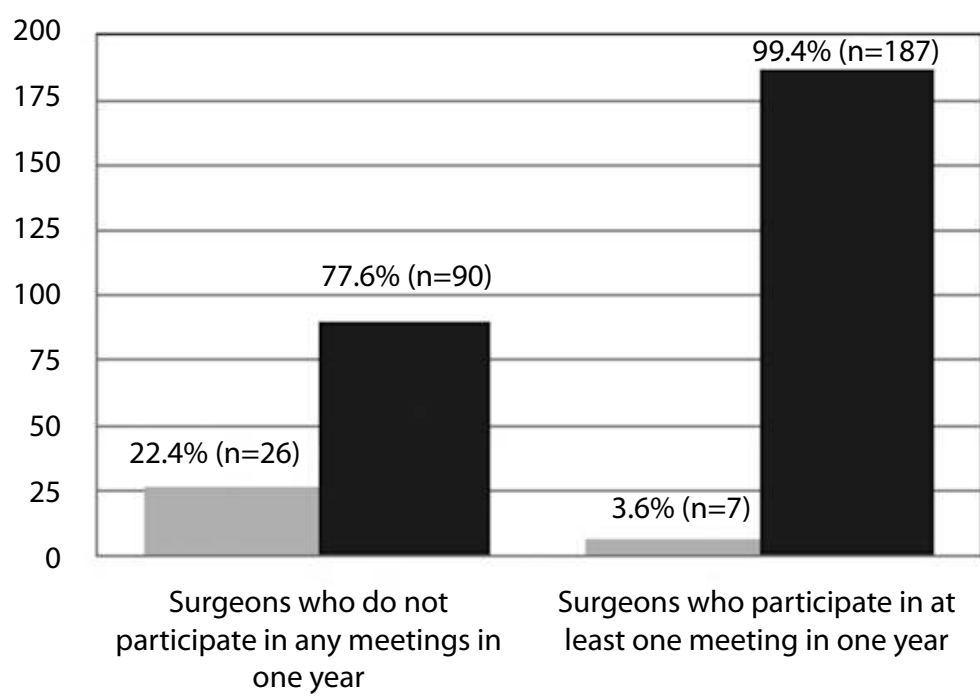

Surgeons who do not

implement nutritional screening

Surgeons who implement nutritional screening

Figure 2. The distribution of the surgeons according to participation in the scientific meetings on clinical nutrition and the implementation of nutritional screening

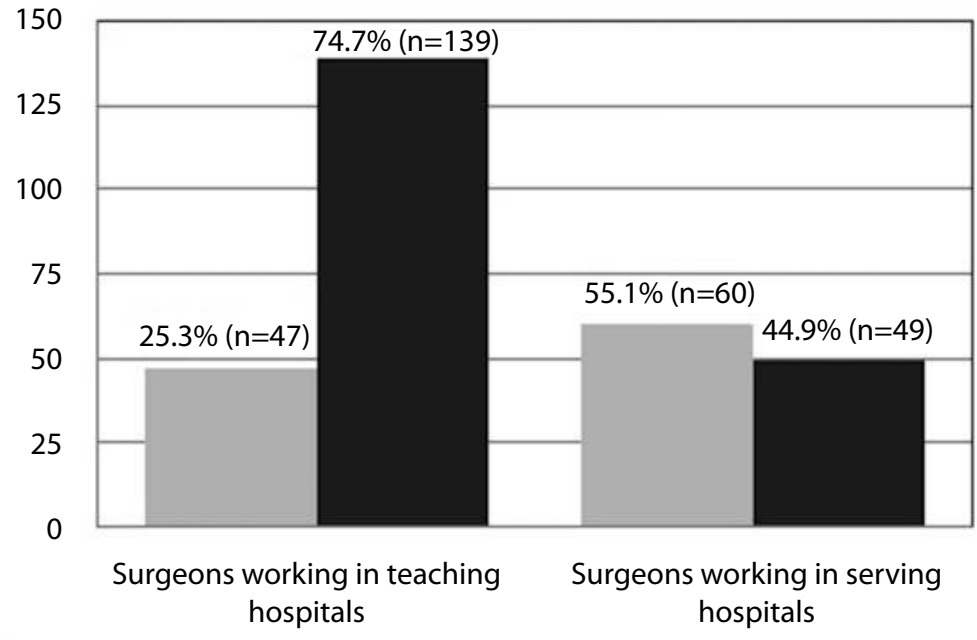

Surgeons who do not participate in scientific meetings

Surgeons who participate in at least one scientific meeting

Figure 3. The distribution of the surgeons according to their affiliation and participation in the scientific meetings on clinical nutrition

Forty-seven of 312 respondents (15.1\%) ceased oral intake of solid food $6 \mathrm{~h}$ before operation. Forty-seven respondents (15.1\%), but not necessarily the same respondents who ceased solid food intake $6 \mathrm{~h}$ before the operation, ceased the oral intake of clear liquids $2 \mathrm{~h}$ before anesthesia induction. Thirty-one participants (9.9\%) ceased the oral intake of both clear liquids and solid food preoperatively, according to the American Society of Anesthesiologists (ASA) guidelines for preoperative fasting (8). The participants who attended at least one scientific meeting on nutrition in a year had a significantly higher tendency to act according to these guidelines $(p<0.001)$. Further, the participants who attended 3 or more meetings had a significantly higher tendency to act according to the guidelines when compared with surgeons attending 1 or 2 meetings $(p<0.001)$.

Sixty-six participants (21.2\%) stated that they preferred immunonutrition products for their patients undergoing major ab- dominal surgery for cancer. Two hundred and forty-four participants stated that they preferred to give standard NS to the patients with severe nutritional risk $(78.2 \%)$, whereas a small ratio of participants preferred not to give any NS $(n=2,0.6 \%)$ to this patient group. A significantly higher number of surgeons participating in scientific meetings on nutrition used immunonutrition in their clinical practice when compared with the surgeons who did not participate in these meetings $(p=0.007)$.

\section{DISCUSSION}

Depending on the diagnostic criteria, the patient population, and the acknowledged definition, in-hospital prevalence of malnutrition is reported to be between $20 \%$ and $50 \%$, and it may even be higher in the surgical wards for certain patient populations $(2,4,9)$. Despite these high rates of malnutrition, nutritional practice was shown to be insufficient due to the lack of knowledge and interest among doctors and nurses 


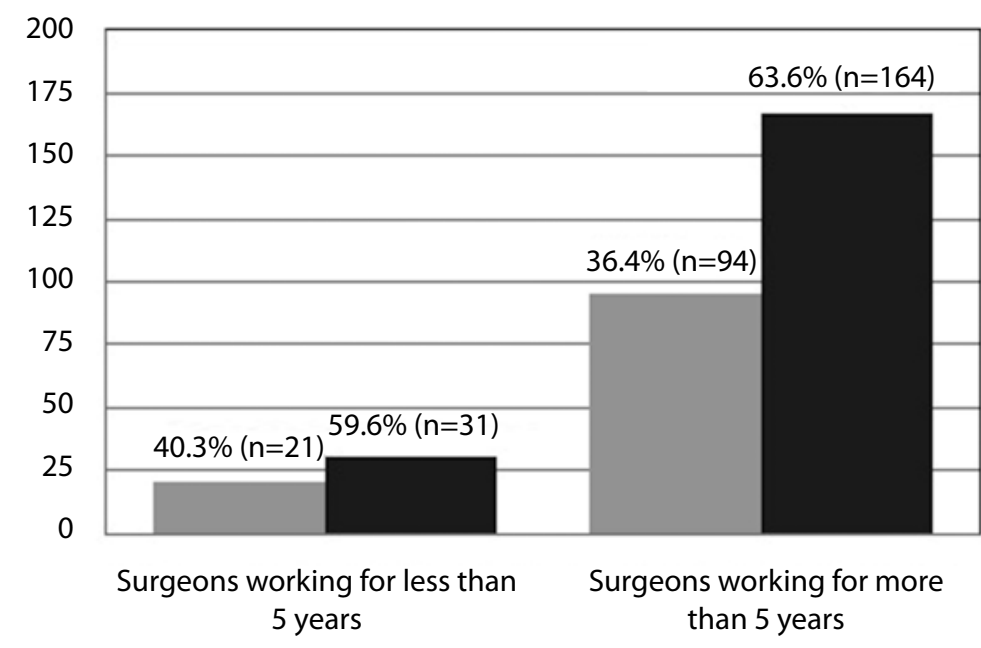

Surgeons who do not participate in scientific meetings

Surgeons who participate in at least one scientific meeting

Figure 4. The distribution of the surgeons according to their experience and participation in the scientific meetings on clinical nutrition

$(10,11)$. Although there are very few studies on the awareness of nutritional topics among surgeons as a specific group of clinicians, present evidence suggests that the knowledge and clinical practices of surgeons may need improvement (57). Two of the three studies on the subject demonstrated that the surgical trainees had an insufficient level of knowledge regarding clinical nutrition $(6,7)$. Another study, which is the only large-scale study on the current practice of general surgeons with regard to clinical nutrition, surveyed the chairmen of the surgery clinics in Switzerland and Austria regarding the current clinical practice at their centers and demonstrated insufficient implementation of nutrition guidelines (5). The aim of the present study was to screen a broad population of general surgeons in Turkey for the awareness of nutritional principles and their clinical practices, as well as to discuss the influence of some potential factors that may affect their tendencies. Our findings demonstrated that most respondents screen the nutritional status of their patients, which suggests a high rate of awareness among these surgeons. The distribution of these participants shows that experience as a surgeon does not affect the surgeons' sensitivity for the nutritional status of their patients. The duration of the experience in surgery also did not seem to affect the surgeons' level of interest toward clinical nutrition in general, as this factor did not influence whether the surgeon attended any scientific meetings on the subject or not. The institutions the surgeons were affiliated with, however, did have a significant influence on the subject of nutritional screening. Surgeons working in teaching hospitals had a significantly higher tendency to screen nutritional status, as well as a significantly higher tendency to attend scientific meetings on clinical nutrition. The findings of the present study also suggest that attending the aforementioned meetings significantly raises the tendency to screen nutritional status.

Only a quarter of the participants, who screened their patients for malnutrition, screened every patient, whereas most undernourished" or the ones who would undergo a major surgery. The reluctance to screen every patient at hospital admission is sure to lead to an under-diagnosis of malnutrition, especially in patients who do not appear undernourished. The mentioned reluctance, however, does not seem to be restricted either to our sample group or to general surgeons. Grass et al. (5) have demonstrated in their study that only $20 \%$ of the participating surgery clinics in Switzerland and Austria performed routine nutritional screening and only $14 \%$ used the nutritional risk score. Further, literature suggests that there is under-recognition of malnutrition worldwide and only a small percentage of malnourished patients receive NS (12-14).

The most commonly used methods for calculating the caloric need were multiplying the weight of the patient by $25-30 \mathrm{kcal} /$ $\mathrm{kg}$ and the Harris-Benedict formula. The high percentage of participants using these methods suggests that most of these surgeons are capable of calculating the energy needs of their patients.

However, responses to the question "when to start nutritional support in the preoperative period" were generally not in accordance to the American Society for Parenteral and Enteral Nutrition (ASPEN) and European Society of Parenteral and Enteral Nutrition (ESPEN) guidelines $(15,16)$. Only a quarter of the participants initiated NS early enough prior to surgery, whereas the timing stated by the rest of the participants to initiate preoperative NS was inappropriate, decreasing the proven potential benefit of preoperative NS. This finding was also in accordance with the only large-scale study on the subject (5). This situation may either be a result of the lack of followup of the guidelines or distrust to some of the suggestions in the clinical guidelines by some surgeons. The latter explanation is supported by an Australian survey of surgeons, which revealed a higher confidence in the surgeons' own judgment than in clinical practice guidelines and other sources of evidence (17). 
Almost all respondents, who gave preoperative NS, sustained the support in the postoperative period, as also suggested by many authors (18-20). It is also encouraging that almost $70 \%$ of participants prescribed oral NS products after hospital discharge to the patients who received perioperative NS. These findings suggest that the postoperative NS these patients receive is acceptable.

Questions 11 and 12 were added to the questionnaire to inquire the opinion of the surgeons on the novel evidencebased approaches for preoperative fasting. Since 1999, American Society of Anesthesiologists recommends the cessation of clear liquids $2 \mathrm{~h}$ and solid food $6 \mathrm{~h}$ before the induction of anesthesia (8). The continuation of oral feeding in the preoperative period according to these recommendations is also a component of the Enhanced Recovery After Surgery (ERAS) Protocol (21). In this study, only $9.9 \%$ of the participants implement the relatively new evidence-based practices in preoperative fasting. However, this tendency of adhering to the traditional approaches does not seem endemic to the Turkish surgeons, as shown by the previously noted low confidence of the general surgeons in clinical practice guidelines (17). There is also evidence that many crucial components of ERAS are not applied in the western world either, and even in centers where ERAS protocol is practiced, there is considerable variation in the compliance to the components of the protocol (22-24). Depending on the data from the present study and the literature, it can be concluded that the widespread implementation of evidence-based perioperative practices including the curtailed preoperative fast needs more time and effort. One way to accomplish this goal may be postgraduate education of surgeons via conferences and scientific meetings. This opinion is supported by the findings of our study, demonstrating that significantly more surgeons attending at least one scientific meeting on nutrition in a year recommend a preoperative fasting protocol according to ASA guidelines. It is also noteworthy that the participants who attend 3 or more meetings in a year have a higher tendency to practice a curtailed preoperative fast when compared with the surgeons who attend 1 or 2 meetings. This finding suggests that these debates and postgraduate education sessions are more effective in changing certain habits when repeated more frequently.

The last question of the survey was about the preference of immunonutrition products. Although there is an ongoing debate on the subject and opposing data in the literature, perioperative use of immune-modulating enteral products in patients undergoing major abdominal cancer surgery independent of the patient's nutritional status is recommended in the current ESPEN guidelines for enteral nutrition $(16,25,26)$. These immunonutrition products are reimbursed by the state in Turkey and one would expect the use of such formulas for appropriate indications. However, the results of the present study demonstrate that the use of immunonutrition is not common among participants, so this subject may need more clarification among Turkish surgeons. It is again noteworthy that the surgeons participating in at least one scientific meeting a year have a significantly higher tendency to use immunonutrition in their clinical practice. This may suggest that postgraduate education on clinical nutrition may affect the views of the surgeons.
The findings of the study reveal many issues in clinical practice that need to be addressed. The reason for inadequate awareness of nutritional principles lies in many different grounds. Although lectures on nutrition are given to medical students in many universities in Turkey as a chapter of general surgery, these lectures are usually overlooked by many students for being difficult to understand and for having little influence in the rating of their marks since general surgery has many other chapters. In addition, many university and teaching hospitals lack regular postgraduate education in clinical nutrition for their residents. Thus, a structured education program is imperative in the surgical residency program and this program must include repetitive lectures on clinical nutrition relating to surgical patients. Spear et al. (7) demonstrated in their study that the surgical trainees achieved better results soon after an interactive education program consisting of two 1-h lectures on intensive care unit nutrition. However, when these trainees were tested 3 months after completing the course, the mean test scores were lower than the immediate post-test scores, although still being significantly higher than the pre-course levels. The fall in the degree of knowledge with time necessitates the repetition of the lectures. The results of the present study also support this suggestion, as shown by the higher tendency of the surgeons who attend more than 2 meetings to behave according to the ASA guidelines with regard to preoperative fasting.

The major limitation of the present study is that the questionnaire which was e-mailed to 1500 surgeons working all over Turkey to represent the attitudes of a broad population was answered by a relatively small proportion of these surgeons. This and the lack of acquiring a randomized sample group make it difficult to generalize the data drawn out of this study to all Turkish surgeons. However, the authors believe that the sample size of this study is still rather large enough to give a sound opinion of the present situation. Also, when interpreting the findings of this study, we may speculate that the surgeons who participated in this questionnaire had a higher average degree of interest in topics related to nutrition, resulting in a positive bias. Thus, it is possible to conclude that a much better level of awareness and knowledge in nutrition must be achieved for Turkish surgeons in general.

To increase the awareness and knowledge of clinical nutrition amongst surgeons, continuous learning throughout the career through conferences, congresses, and courses seems essential as suggested by the findings of the present survey. Turkish Society of Parenteral and Enteral Nutrition is very active in taking steps to improve nutritional awareness by holding Life Long Learning ${ }^{\circledR}$ courses of ESPEN in Turkish and independent nutrition courses specifically for topics concerning general surgeons as well as for other disciplines in medicine.

\section{CONCLUSION}

The awareness and knowledge of clinical nutrition amongst surgeons needs improving. The findings of the present survey indicate that continuous learning throughout the career through conferences, congresses, and courses is essential to achieve this goal. 
Ethics Committee Approval: Authors declared that the research was conducted according to the principles of the World Medical Association Declaration of Helsinki "Ethical Principles for Medical Research Involving Human Subjects" (amended in October 2013).

Informed Consent: Written informed consent was obtained from surgeons who participated in this study.

Peer-review: Externally peer-reviewed.

Author Contributions: Concept - B.O.B., R.H.G.; Design - B.O.B., S.A., T.S., P.E.E.; Supervision - R.H.G.; Resource - R.H.G., B.O.B., P.E.E.; Materials - R.H.G., B.O.B., P.E.E.; Data Collection and/or Processing - B.O.B., S.A., T.S.; Analysis and/or Interpretation - B.O.B., R.H.G., P.E.E.; Literature Search - B.O.B., S.A., T.S.; Writing - Manuscript - B.O.B., S.A., T.S.; Critical Reviews - R.H.G., P.E.E.

Acknowledgements: The authors kindly thank Jale Karakaya, PhD from Hacettepe University, Department of Biostatistics, for the help she provided in statistical calculations.

Conflict of Interest: No conflict of interest was declared by the authors.

Financial Disclosure: The authors declared that this study has received no financial support.

\section{REFERENCES}

1. Sungurtekin $H$, Sungurtekin $U$, Balci C, Zencir M, Erdem E. The influence of nutritional status on complications after major intraabdominal surgery. J Am Coll Nutr 2004; 23: 227-232. [CrossRef]

2. Norman K, Pichard C, Lochs H, Pirlich M. Prognostic impact of disease-related malnutrition. Clin Nutr 2008; 27: 5-15. [CrossRef]

3. Haldun G, Eren E, Recep A, Hakan K, Mehmet O, Vedat O, et al. Evaluation of nutritional risk on admission to general surgery department. Bratisl Lek Listy 2008; 109: 57-60.

4. Kuzu MA, Terzioglu $H$, Genc V, Erkek AB, Ozben M, Sonyürek P, et al. Preoperative nutritional risk assessment in predicting postoperative outcome in patients undergoing major surgery. World $\mathrm{J}$ Surg 2006; 30: 378-390.

5. Grass F, Cerantola Y, Schäfer M, Müller S, Demartines N, Hübner M. Perioperative nutrition is still a surgical orphan: results of a SwissAustrian survey. Eur J Clin Nutr 2011; 65: 642-647. [CrossRef]

6. Awad S, Herrod PJ, Forbes E, Lobo DN. Knowledge and attitudes of surgical trainees towards nutritional support: food for thought. Clin Nutr 2010; 29: 243-248. [CrossRef]

7. Spear S, Sim V, Moore FA, Todd SR. Just Say No to Intensive Care Starvation: A Nutrition Education Program for Surgery Residents. Nutr Clin Pract 2013; 28: 387-391. [CrossRef]

8. Practice guidelines for preoperative fasting and the use of pharmacologic agents to reduce the risk of pulmonary aspiration: application to healthy patients undergoing elective procedures: a report by the American Society of Anesthesiologist Task Force on Preoperative Fasting. Anesthesiology 1999; 90: 896-905. [CrossRef]

9. Barker LA, Gout BS, Crowe TC. Hospital malnutrition: prevalence, identification and impact on patients and the healthcare system. Int J Environ Res Public Health 2011; 8: 514-527. [CrossRef]

10. Mowe M, Bosaeus I, Rasmussen HH, Kondrup J, Unosson M, Irtun $\varnothing$. Nutritional routines and attitudes among doctors and nurses in Scandinavia: a questionnaire based survey. Clin Nutr 2006; 25 : 524-532. [CrossRef]

11. Mowe M, Bosaeus I, Rasmussen HH, Kondrup J, Unosson M, Rothenberg $E$, et al. Insufficient nutritional knowledge among health care workers? Clin Nutr 2008; 27: 196-202. [CrossRef]

12. Correia MI, Campos AC, ELAN Cooperative Study. Prevalence of hospital malnutrition in Latin America: the multicenter ELAN study. Nutrition 2003; 19: 823-825. [CrossRef]

13. McWhirter JP, Pennington CR. Incidence and recognition of malnutrition in hospital. BMJ 1994; 308: 945-948. [CrossRef]

14. Rasmussen $\mathrm{HH}$, Kondrup J, Staun M, Ladefoged K, Kristensen $\mathrm{H}$, Wengler A. Prevalence of patients at nutritional risk in Danish hospitals. Clin Nutr 2004; 23: 1009-1015. [CrossRef]

15. ASPEN Board of Directors and the Clinical Guidelines Task Force. Guidelines for the use of parenteral and enteral nutrition in adult and pediatric patients. JPEN J Parenter Enteral Nutr 2002; 26: 1SA138SA.

16. Weimann $A$, Braga $M$, Harsanyi L, Laviano A, Ljungqvist $O$, Soeters $P$, et al. ESPEN Guidelines on Enteral Nutrition: Surgery including Organ Transplantation. Clin Nutr 2006; 25: 224-244. [CrossRef]

17. Kitto S, Villanueva EV, Chesters J, Petrovic A, Waxman BP, Smith JA. Surgeons' attitudes towards and usage of evidence-based medicine in surgical practice: a pilot study. ANZ J Surg 2007; 77: 231-236. [CrossRef]

18. Keele AM, Bray MJ, Emery PW, Duncan HD, Silk DB. Two phase randomised controlled clinical trial of postoperative oral dietary supplements in surgical patients. Gut 1997; 40: 393-399. [CrossRef]

19. Beattie AH, Prach AT, Baxter JP, Pennington CR. A randomised controlled trial evaluating the use of enteral nutritional supplements postoperatively in malnourished surgical patients. Gut 2000; 46: 813-818. [CrossRef]

20. Smedley F, Bowling T, James M, Stokes E, Goodger C, O'Conner O, et al. Randomized clinical trial of the effects of preoperative and postoperative oral nutritional supplements on clinical course and cost of care. Br J Surg 2004; 91: 983-990. [CrossRef]

21. Lassen K, Soop M, Nygren J, Cox PB, Hendry PO, Spies C, et al. Consensus review of optimal perioperative care in colorectal surgery: Enhanced Recovery After Surgery (ERAS) Group recommendations. Arch Surg 2009; 144: 961-969. [CrossRef]

22. Kehlet $H$, Büchler MW, Beart RW Jr, Billingham RP, Williamson R. Care After Colonic Operation- Is it Evidence Based? Results from a Multinational Survey in Europe and the United States. J Am Coll Surg 2006; 202: 45-54. [CrossRef]

23. Lassen $\mathrm{K}$, Hannemann $\mathrm{P}$, Ljungqvist $\mathrm{O}$, Fearon $\mathrm{K}$, Dejong $\mathrm{CH}$, von Meyenfeldt MF, et al. Patterns in current perioperative practice: survey of colorectal surgeons in five northern European countries. BMJ 2005; 330: 1420-1421. [CrossRef]

24. Ahmed J, Khan S, Lim M, Chandrasekaran TV, Mac Fie J. Enhanced recovery after surgery protocols - compliance and variation in practice during routine colorectal surgery. Colorectal Dis 2012; 14: 1045-1051. [CrossRef]

25. Mc Cowen KC, Bistrian BR. Immunonutrition: problematic or problem solving? Am J Clin Nutr 2003; 77: 764-770.

26. Marimuthu K, Varadhan KK, Ljungqvist O, Lobo DN. A Meta-Analysis of the Effect of Combinations of Immune Modulating Nutrients on Outcome in Patients Undergoing Major Open Gastrointestinal Surgery. Ann Surg 2012; 255: 1060-1068. [CrossRef] 


\section{APPENDIX}

The questionnaire for the survey to define the approaches of the Turkish surgeons toward nutritional screening and therapy of surgical patients.

1. How long have you been working as a surgeon?
a. Less than 5 years

b. More than 5 years

2. What type of an institution are you working with?
a. A teaching hospital (University or Training and Re- search Hospital affiliated to the Ministry of Health)
b. General hospital (State Hospital affiliated to the Minis- try of Health)

3. How many meetings on clinical nutrition do you participate in in a year?
a. None
b. 1-2
c. More than 2

4. Do you screen the nutritional status of your hospitalized patients?
a. Yes
b. No

5. Which of your patients do you screen for malnutrition?
a. All my patients
b. The ones who appear undernourished by inspection
c. The ones who will be undergoing a major operation

6. Which system do you use for nutrition screening?
a. Nutritional risk screening - 2002
b. Subjective Global Assessment
c. Laboratory Tests
d. Anthropometric Tests
e. I use multiple methods to determine the nutritional risk in the same patient

7. How many days prior to the operation do you start nutritional support in a patient with a high nutrition risk?
a. 3-4
b. $5-7$
c. $10-14$

8. How do you calculate the daily energy requirement of your patients?

a. I use Harris-Benedict formula

b. I give $25-30 \mathrm{kcal} / \mathrm{kg}$ per day depending on the clinical situation
c. I use another formula
d. I do not calculate it myself, I consult to a dietitian

9. To a patient whom I have given nutritional support before the operation,

a. I do not give postoperative nutritional support

b. I always give postoperative nutritional support

c. I give postoperative nutritional support if the patient will not be able to fulfill his nutritional needs by oral food intake.

10. If you have given nutritional support to your patient during hospital stay, do you prescribe nutritional support products to him/her when discharging from the hospital?
a. Yes, for 2 weeks
b. Yes, for 4-6 weeks
c. No

11. When do you cease the oral intake of solid food in a patient who will be undergoing a major abdominal operation?
a. $12 \mathrm{~h}$ before the operation
b. At midnight before the operation day
c. $6 \mathrm{~h}$ before the operation

12. When do you cease the oral intake of clear fluids in a patient who will be undergoing a major abdominal operation?
a. $6 \mathrm{~h}$ before the operation
b. At midnight before the operation day
c. $2 \mathrm{~h}$ before the operation

13. To a patient who will undergo a major abdominal operation for cancer,
a. I do not give nutritional support
b. I give standard nutritional support products to the pa- tients at nutritional risk
c. I give immunonutrition products regardless of the nu- tritional risk

Since this survey was planned with the intention of defining the tendencies of general surgeons regarding nutritional screening and therapy, some of the questions do not have any right or wrong answers. However, the desirable answers for the questions 4, 5, 7, 10, 11, and 12 are $a, a, c, b, c$, and c, respectively. For the 13 th question, both $b$ and $c$ are acceptable. 